\title{
A switch-on molecular biosensor for detection of caspase-3 and imaging of apoptosis of cells
}

\author{
Rui Gong ${ }^{1,2}$, Dianbing Wang ${ }^{1}$, Ghulam Abbas ${ }^{1,2}$, Shimin Li $^{1,2}$, Qian Liu ${ }^{1}$, Mengmeng Cui ${ }^{1} \&$ \\ Xian-En Zhang ${ }^{1,2,3 *}$ \\ ${ }^{1}$ National Laboratory of Biomacromolecules, CAS Center for Excellence in Biomacromolecules, Institute of Biophysics, Chinese Academy of \\ Sciences, Beijing 100101, China; \\ ${ }^{2}$ University of Chinese Academy of Sciences, Beijing 100049, China; \\ ${ }^{3}$ Faculty of Synthetic Biology, Shenzhen Institute of Advanced Technology, Chinese Academy of Sciences, Shenzhen 518055, China
}

Received June 15, 2021; accepted July 26, 2021; published online September 9, 2021

\begin{abstract}
Apoptosis is a form of programmed cell death that is essential for maintaining internal environmental stability. Disordered apoptosis can cause a variety of diseases; therefore, sensing apoptosis can provide help in study of mechanism of the relevant diseases and drug development. It is known that caspase-3 is a key enzyme involved in apoptosis and the expression of its activity is an indication of apoptosis. Here, we present a genetically encoded switch-on mNeonGreen2-based molecular biosensor. mNeonGreen 2 is the brightest monomeric green fluorescent protein. The substrate of caspase-3, DEVD amino acid residues, is inserted in it, while cyclized by insertion of Nostoc punctiforme DnaE intein to abolish the fluorescence (inactive state). Caspase3-catalyzed cleavage of DEVD linearizes mNeonGreen2 and rebuilds the natural barrel structure to restore the fluorescence (activated state). The characterization exhibited that the Caspase-3 biosensor has shortened response time, higher sensitivity, and prolonged functional shelf life in detection of caspase- 3 amongst the existing counterparts. We also used the Caspase- 3 biosensor to evaluate the effect of several drugs on the induction of apoptosis of HeLa and MCF-7 tumor cells and inhibition of Zika virus invasion.
\end{abstract}

apoptosis, caspase-3, molecular biosensor, molecular imaging, Zika virus

Citation: Gong, R., Wang, D., Abbas, G., Li, S., Liu, Q., Cui, M., and Zhang, X.E. (2022). A switch-on molecular biosensor for detection of caspase-3 and imaging of apoptosis of cells. Sci China Life Sci 65, 540-549. https://doi.org/10.1007/s11427-021-1986-7

\section{INTRODUCTION}

Apoptosis, or programmed cell death, is a fundamental feature of all animal cells which is involved in cell growth, division, and differentiation, and causes a series of changes in morphology and metabolism (Jacobson et al., 1997). The dysregulation of apoptosis can cause many diseases, such as Alzheimer's disease, Huntington's disease, and autoimmune disorders along with immortality of cancer cells (Cotter, 2009; Nijhawan et al., 2000; Stefanis et al., 2001). Under-

*Corresponding author (email: zhangxe@ibp.ac.cn) standing the physiological mechanisms of apoptosis in living cells is crucial for the diagnosis and treatment of relevant diseases. Conventionally, morphological and biochemical methods are used for apoptosis detection, but these are not sufficient and accurate enough to reflect the in-depth apoptotic mechanisms (Galluzzi et al., 2012; Hengartner, 2000). Proteomics is capable of identifying the caspase substrates in apoptosis (Pham et al., 2012), but it is lagging.

In recent years, caspases are found highly associated with cell apoptosis. Among these, caspase- 3 is a common downstream effector of apoptotic pathways, which can transmit and amplify apoptotic signals (Porter and Jänicke, 1999) and 
can therefore be used as the analytical target to represent apoptosis. The detection of Caspase-3 biosensor is based on the hydrolytic activity of its short peptide DEVD. In Förster/ fluorescence resonance energy transfer (FRET), cyan fluorescent protein (CFP) and yellow fluorescent protein (YFP) are covalently linked by DEVD. If the linker is intact, YFP emission $(525 \mathrm{~nm})$ can be induced by CFP excitation. If the linker is cleaved by caspase-3, FRET is abolished, causing CFP emission (475 nm) (Valentin et al., 2005). Green fluorescent protein (GFP)-DEVD-quencher structure is also designed to be sensitive to caspase-3. Cleavage of the DEVD sequence leads to the restoration of GFP fluorescence, indicating the existence of caspase-3 (Nicholls et al., 2011). Another strategy is the use of luciferase. Luciferase was cyclized by insertion of DnaE intein and DEVD at different sites. Cleavage of the DEVD sequence by caspase-3 restored the linear structure of luciferase and rescued its enzyme activity (Kanno et al., 2009; Kanno et al., 2007). Moreover, the spatial translocation-based fluorescent sensors (Bardet et al., 2008) and self-assembly-based fluorescent sensors (Nasu et al., 2016; Zhang et al., 2013; Zhang et al., 2019) have been developed. These methods have been used to study apoptosis but possess many drawbacks. In luciferase assay, exogenous addition of enzyme substrate is required to generate catalytical fluorescence. Although it can produce higher signal intensity, it has the potential risk of interfering with the normal physiological processes (Kanno et al., 2007). The endogenous genetically encoded fluorescent protein biosensors overcome these problems, but may have some other limitations, such as lower sensitivity and the delayed response time due to fluorescence maturation (Xu et al., 2020).

Here, to build a sensitive and endogenous molecular sensor for the detection of caspase- 3 and apoptosis, we propose mNeonGreen 2 as a sensing element that is the brightest monomeric fluorescent protein (1.5 to 3 times higher than that of GFP and YFP) with a shorter maturation time among the GFP originated fluorescent proteins (Shaner et al., 2013). The molecular biosensor is based on turning the inactive state (no fluorescence emission) to the active state (emission) of $\mathrm{mNeonGreen} 2$ (Figure 1A). The mNeonGreen2 protein is initially fused with the caspase-3 cleavage site DEVD and cyclized by insertion of Nostoc punctiforme DnaE intein (Npu DnaE) (Guerreiro et al., 2019) at appropriate site to abolish its fluorescence. In the presence of caspase-3, DEVD is cleaved allowing linearization and undergo confirmational changes to reform the beta-barrel structure of mNeonGreen2 to restore the fluorescence emission.

\section{RESULTS}

\section{Generation and optimization of the Caspase-3 biosensor}

We selected mNeonGreen 2 as the sensing element in this study. To obtain an effective genetically encoded biosensor for caspase-3 activity detection, we performed a protein splicing reaction utilizing $N p u$ DnaE and DEVD, the cleavage site of caspase- 3 . The cyclized fluorescent protein mNeonGreen2 lost its fluorescence activity on distortion of its structure. The activity of the $\mathrm{mNeonGreen} 2$ protein was restored once the cleavage site was digested by caspase- 3 and the protein was converted to a linear form (Figure 1A). At high concentrations of the biosensor, the $\mathrm{N}$-terminal of one biosensor could complement the C-terminal of another to produce a significant background fluorescence. To reduce the background fluorescence, the PEST sequence, a proteolytic signal (Rechsteiner and Rogers, 1996), was attached to the C-terminal of the biosensor. The unspliced biosensor containing the PEST sequence reduced background fluorescence on its degradation (Kanno et al., 2007). The circularly permuted mNeonGreen2 biosensor was constructed with newly generated N- and C-terminal ends at residues T144/ Y145, D158/K159, and N173/G174 with DnaEc and DnaEn fragments as shown in Table S1 in Supporting Information. Transiently transfected 293T cells with the three aforementioned biosensors were cultured for $18 \mathrm{~h}$, and then stimulated with staurosporine (STS), an apoptosis-inducing reagent, which triggers the digestion of the DEVD amino acid sequence by caspase-3. Images were captured and analyzed using Cytation-3 every $2 \mathrm{~h}$. Almost all the cells with the N173/G174 biosensor showed bright fluorescence before stimulation, which was not dependent on the apoptosis. The fluorescence of the T144/Y145 biosensor was restored, but a long maturation time $(15 \mathrm{~h})$ was necessary for the restoration. The D158/K159 biosensor was deemed as the best for caspase- 3 detection, with a short maturation time and reliable response compared with the T144/Y145 and N173/G174 biosensors (Figure 1B).

To obtain a robust biosensor, we truncated the two ends of the D158/K159 biosensor and found that the deletion of the C-terminal residues 230-238 did not significantly affect fluorescence compared with the full-length construct, but the length of the N-terminal residues significantly affected fluorescence intensity and maturation time. We created a library of candidate constructs D0-D5 based on the deletion of the N-terminal while maintaining the $\beta$-barrel structure of mNeonGreen 2 intact (Table S1 in Supporting Information). The fluorescence of the 293T cells transiently expressing the biosensor candidates was detected after treatment with $100 \mathrm{nmol} \mathrm{L}^{-1}$ STS every $2 \mathrm{~h}$. We also found that the response time of these biosensors showed significant differences. The full-length mNeonGreen 2 biosensor needed about $15 \mathrm{~h}$ for maturation. The D1 and D2 constructs, obtained by deletion of the N-terminal residues 1-4 or 1-6, respectively, and Cterminal residues $230-238$ of the $\beta$-barrel structure, only needed $8 \mathrm{~h}$ for maturation, and the D2 construct with a shorter response time and weaker background fluorescence 

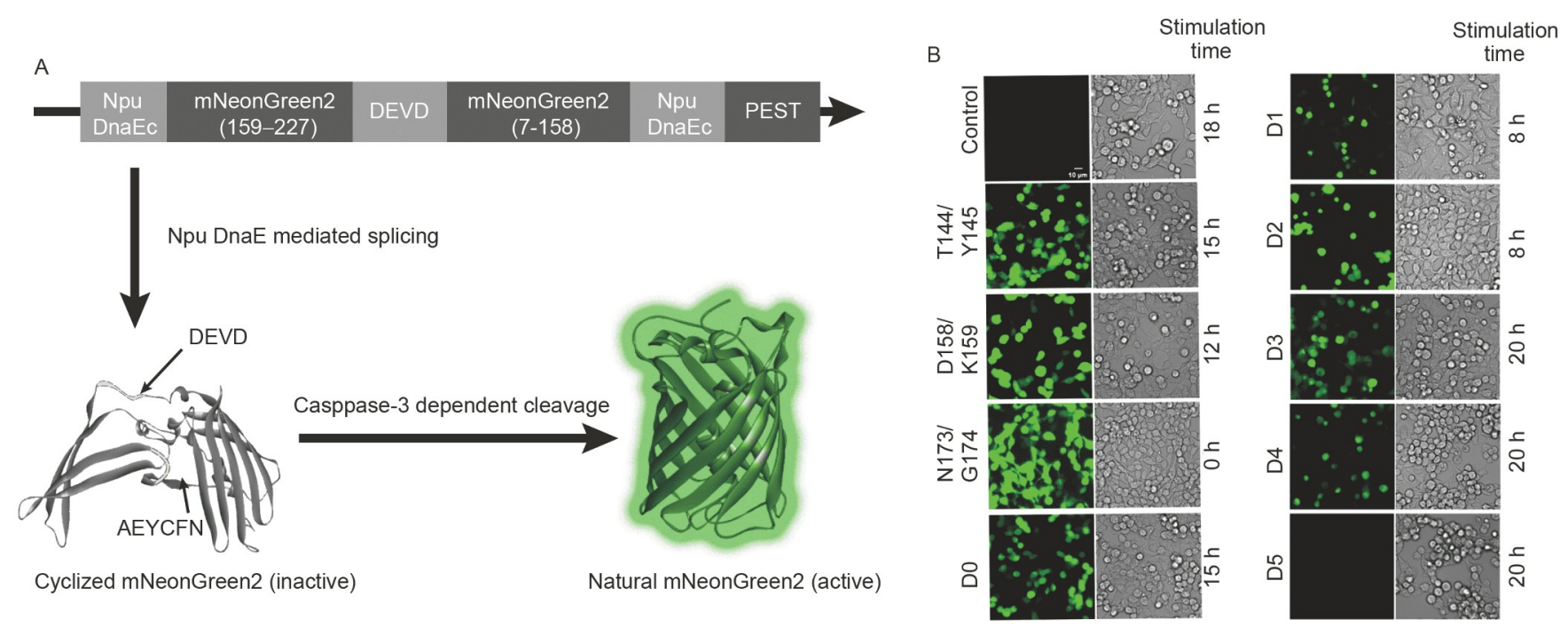

Figure 1 Generation and optimization of Caspase-3 biosensors. A, Principle of the mNeonGreen2-based biosensor. B, The fluorescence of candidates was detected after treatment with $100 \mathrm{nmol} \mathrm{L}^{-1}$ STS for an opportune time. Control is the 293T cells without biosensor. T144/Y145, D158/K159, N173/G174, and D0-D5 are according to Table S1 in Supporting Information.

was selected as our Caspase-3 biosensor (Figure 1B). This biosensor could reduce the background fluorescence to the greatest extent and mature more efficiently, which is crucial for the detection of caspase-3 activity. Subsequent deletion of the amino acids at the N-terminals of the mNeonGreen2 protein led to less background fluorescence (D3, D4), but needed more time for maturation. The deletion of amino acid residues $1-14$ at the $\mathrm{N}$-terminal influenced the stability of the fluorescent protein and no fluorescence was detected; therefore, D5 could not reflect the activity of caspase-3.

\section{Verification and characterization of the Caspase- 3 bio- sensor}

To assess the feasibility of the biosensor, we generated a control named CK biosensor for the Caspase-3 biosensor replacing DEVD with GSGC, which is not digested by active caspase-3. We stably expressed Caspase-3 biosensor and CK biosensor in the 293T cells and carried out stimulation with STS or the caspase-3 inhibitor Z-VAD-fmk or with both STS and Z-VAD-fmk for $10 \mathrm{~h}$. We detected fluorescence-positive 293T cells without STS, which means that programmed cell death (apoptosis) is a normal process. Stimulation with STS induced a strong increase in the fluorescence at a concentration of $100 \mathrm{nmol} \mathrm{L}^{-1}$, and $100 \mu \mathrm{mol} \mathrm{L}{ }^{-1}$ of Z-VAD-fmk completely suppressed the activity of caspase-3. The GSGC sequence replacing DEVD was not digested by caspase-3, and no fluorescence was detected under the same detection parameters (Figure 2A). These results indicated that cells stably expressing Caspase-3 biosensor can be used to indicate apoptosis in living cells. Interestingly, we observed apoptosis in the cells after stimulation with STS (Figure 2B). Figure $2 \mathrm{~B}$ shows that the Caspase- 3 biosensor required about
$80 \mathrm{~min}$ for its activation, which is similar to previously reported times of 70-90 min (Zhang et al., 2013), and the apoptotic bodies were produced with the fluorescence increased. The fluorescence appeared at $20 \mathrm{~min}$ whereas the shedding of the membranous microvesicles from the cells at about $80 \mathrm{~min}$, which means that our biosensor detects apoptosis earlier than the production of apoptotic bodies.

To quantitatively evaluate the effect of STS on caspase- 3 activity, we defined a relative fluorescence unit (RFU), where the fluorescence intensity from different concentrations of STS was divided by that obtained from $0 \mathrm{mmol} \mathrm{L}^{-1}$ STS. The 293T (Caspase-3 biosensor) cells were stimulated with different concentrations of STS for $10 \mathrm{~h}$. The results showed that the fluorescence intensity increased with the increasing concentration of STS. Treatment with 100 and $500 \mathrm{nmol} \mathrm{L}^{-1}$ of STS had similar effects, but $1 \mathrm{mmol} \mathrm{L}^{-1}$ of STS caused intense cell death, and no fluorescence was detected (data not shown). Cleavage of the Caspase-3 biosensor was completely suppressed by $100 \mu \mathrm{mol} \mathrm{L}^{-1}$ of Z-VAD-fmk (Figure 2C). In accordance with the caspase-3 activity assay based on the fluorescence intensity, Western blots confirmed the upregulation of cleaved caspase-3 and cleaved Caspase-3 biosensor (Figure S1 in Supporting Information). The results are consistent with cyclic Fluc sensor previously reported by Kanno et al. in 2007 (Kanno et al., 2007). Our biosensor can detect the fluorescence in situ but it needs a long maturation time, while the Luciferase sensor is faster but the cells need to be harvested first and then the lysates should be mixed with a Fluc substrate, which increases the complexity of the experiment (Kanno et al., 2007).

Drug action is an ongoing process, so it is crucial to understand the dynamic process of the curve profile of maturation and quenching after stimulation. Therefore, we 
A
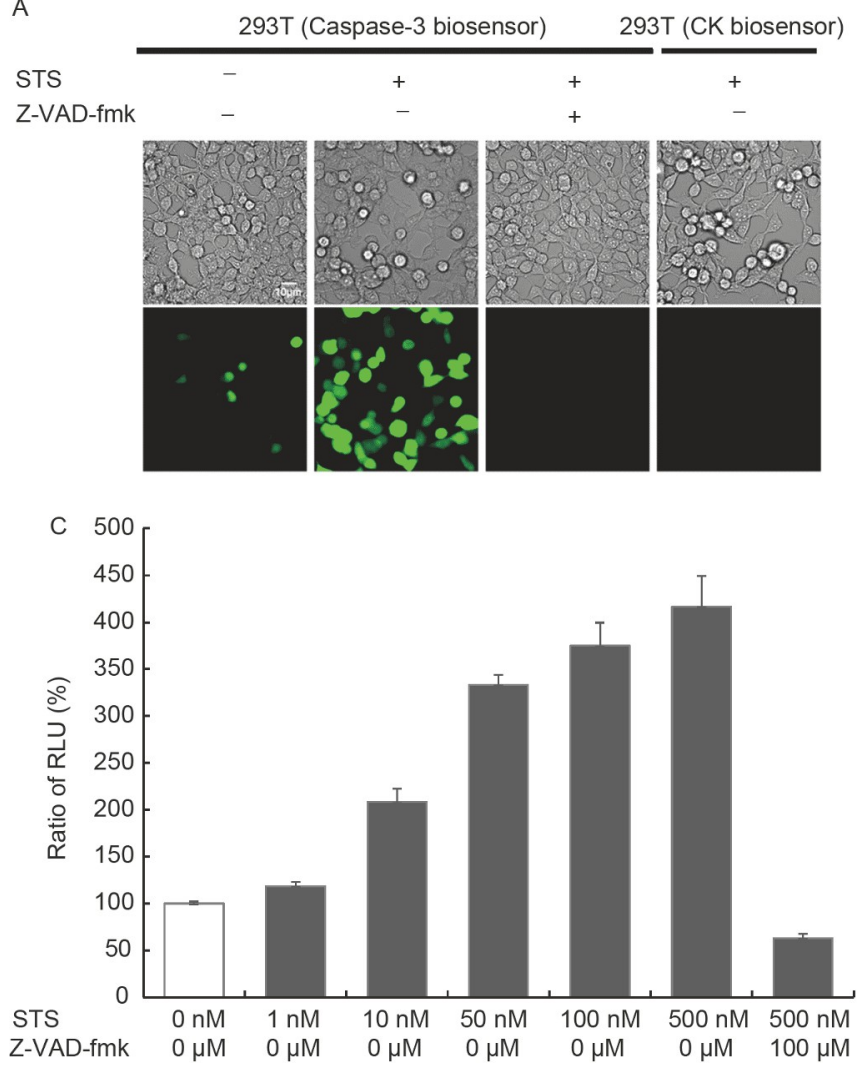

B

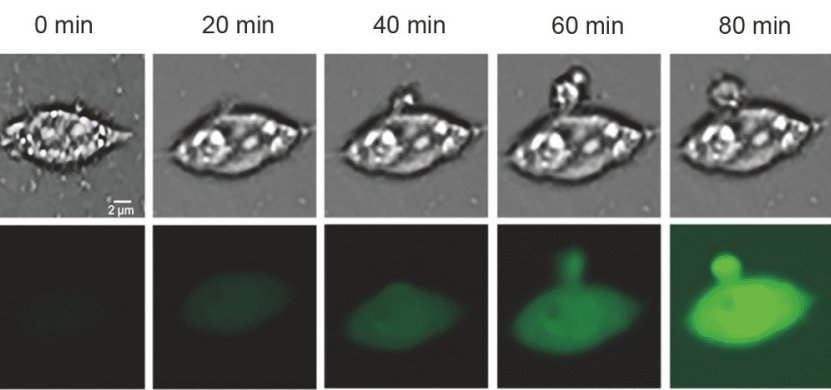

$\mathrm{D}$

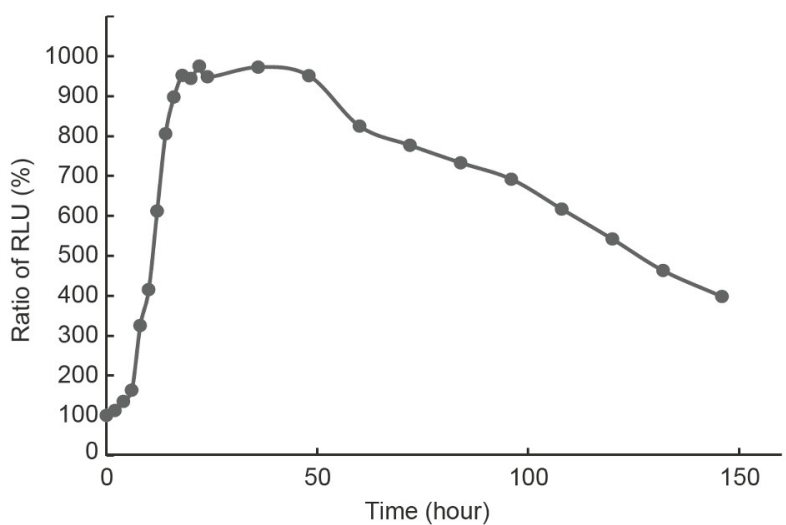

Figure 2 Verification and characterization of the biosensor. A, The fluorescence of 293T (Caspase-3 biosensor) and 293T (CK biosensor) cells after treatment with $500 \mathrm{nmol} \mathrm{L}^{-1}$ STS or control for $10 \mathrm{~h}$ in the presence or absence of $100 \mu \mathrm{mol} \mathrm{L}{ }^{-1} \mathrm{Z}$-VAD-fmk. B, The emergence of fluorescence in cell was detected. C, Quantitative analysis of the biosensor after stimulation with various concentrations of STS or $100 \mu \mathrm{mol} \mathrm{L}^{-1} \mathrm{Z}$-VAD-fmk for $10 \mathrm{~h}$. D, Fluorescence intensity of 293T (Caspase-3 biosensor) cells versus time after treatment with $100 \mathrm{nmol} \mathrm{L}^{-1}$ STS.

performed real-time analysis of caspase-3 activity in $293 \mathrm{~T}$ cells. The fluorescence intensity was detected in the 96-well plate every $2 \mathrm{~h}$ after stimulation with $100 \mathrm{nmol} \mathrm{L}^{-1}$ of STS, and then detected every $12 \mathrm{~h}$ after maturation. The relative fluorescence unit (RFU) was now defined as the fluorescence intensity with different times divided by that obtained from $0 \mathrm{~h}$. The fluorescence intensity initially showed a slow increase for $6 \mathrm{~h}$; thereafter, a rapid increase was observed and it reached a maximum saturation after $18 \mathrm{~h}$. The half-life (the time required for the fluorescence intensity to reach half of the maximum) of the cleaved Caspase- 3 biosensor was more than $100 \mathrm{~h}$ which provides a sufficient time for highthroughput screening of chemical compounds (Figure 2D). From the above results, we believe that Caspase-3 biosensor is a reliable biosensor for the quantitative analysis of apoptosis-induced cell death.

\section{The Caspase-3 biosensor accurately indicated TNF- $\alpha$ - induced apoptosis in tumor cells}

The practicality of the Caspase- 3 biosensor also needed to be tested; therefore, we stably expressed Caspase- 3 biosensor with the tumor cells HeLa and MCF-7 and examined whether the activity of the cyclic Caspase-3 biosensor was restored after stimulation with TNF- $\alpha$. It is worth noting that MCF-7 cells are deficient in caspase-3, while caspase-7, a caspase-3like DEVDase is present (Zhang et al., 2013). TNF- $\alpha$ is a small protein secreted by macrophages and is used as an antineoplastic drug. In recent years, some studies have used TNF- $\alpha$ in clinical trials, and the direct inhibition of TNF- $\alpha$ has become a major treatment option for autoimmune diseases (Conrad et al., 2018; Martin et al., 2019). Here, we detected the apoptosis of tumor cells HeLa and MCF-7 induced by TNF- $\alpha$. The tumor cells that stably expressed Caspase- 3 biosensor were treated with $50 \mathrm{ng} \mathrm{mL}^{-1}$ of TNF- $\alpha$, $10 \mu \mathrm{g} \mathrm{mL}^{-1}$ of cycloheximide (CHX), or both, and the fluorescence was detected after $10 \mathrm{~h}$. No fluorescence was detected in the control cells without Caspase- 3 biosensor, and the HeLa (Caspase-3 biosensor) cells without stimulation showed negligible fluorescence. Both $\mathrm{CHX}$ and TNF- $\alpha$ could induce apoptosis. TNF- $\alpha$ and co-treatment with CHX could significantly trigger cell death and release a strong fluorescence, which is consistent with previous reports (Hellwig et al., 2008). As expected, the fluorescence was blocked by Z-VAD-fmk, despite the presence or absence of TNF- $\alpha$ and CHX (Figure 3A; Figure S2 in Supporting In- 
A

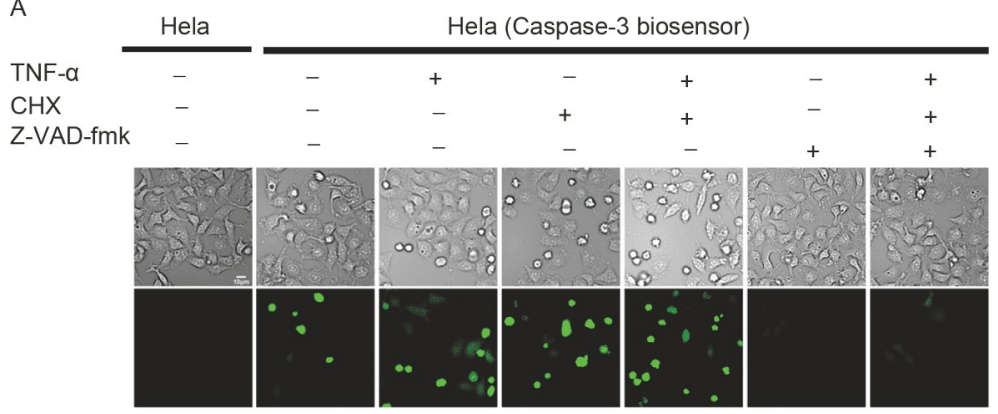

B

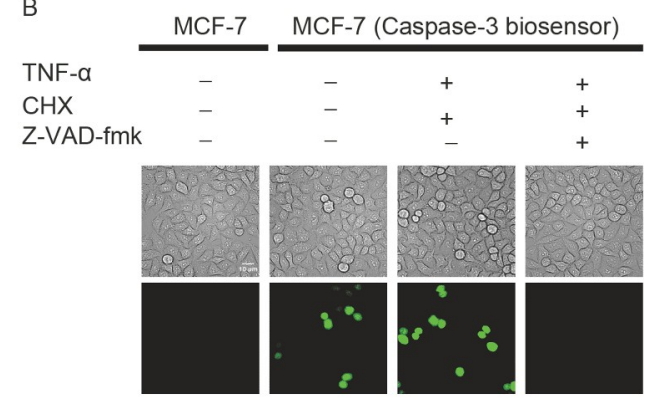

Figure 3 Caspase-3 biosensor characterization in response to TNF- $\alpha$. A, The fluorescence images of HeLa (Caspase-3 biosensor) cells were detected after treatment with $50 \mathrm{ng} \mathrm{mL}^{-1}$ of TNF- $\alpha$ or $10 \mu \mathrm{g} \mathrm{mL}^{-1}$ cycloheximide (CHX) or both of them or control for $10 \mathrm{~h}$ in the presence or absence of $100 \mu \mathrm{mol} \mathrm{L} \mathrm{L}^{-1} \mathrm{Z}$ VAD-fmk. B, The fluorescence images of MCF-7 (Caspase-3 biosensor) cells were captured after treatment with $50 \mathrm{ng} \mathrm{mL}^{-1}$ of TNF- $\alpha$ and $10 \mu \mathrm{g} \mathrm{mL}$ cycloheximide (CHX) or control for $10 \mathrm{~h}$ in the presence or absence of $100 \mu \mathrm{mol} \mathrm{L}{ }^{-1} \mathrm{Z}$-VAD-fmk.

formation). Similar results were also obtained for the MCF-7 (Caspase-3 biosensor) cells, but the apoptosis was slightly lesser than that seen in the HeLa cells (Figure 3B; Figure S2 in Supporting Information). These results reconfirm that the Caspase-3 biosensor could accurately reflect DEVDase-dependent cell death.

\section{The Caspase-3 biosensor indicates the apoptosis of tu-} mor cells after stimulation with chemotherapeutic drugs

We tested three types of antitumor drugs: 5-fluorouracil (5FU), which influences the synthesis of nucleic acids (Sara et al., 2018); docetaxel, which obstructs the synthesis of proteins (Sun et al., 2018); and adriamycin, which inhibits the synthesis of RNA and DNA (Blum and Carter, 1974). The concentration of drugs was based on previous reports and considerations of their clinical use. The HeLa (Caspase-3 biosensor) and MCF-7 (Caspase-3 biosensor) cells were stimulated with different concentrations of 5-FU $(0,10,100$, and $\left.1,000 \mu \mathrm{mol} \mathrm{L}^{-1}\right)$ for $48 \mathrm{~h}$. 5-FU showed a better killing effect on the HeLa (Caspase-3 biosensor) cells (Figure 4A), but no killing effect was detected in the MCF-7 cells (Figure S3 in Supporting Information). The low concentration (10 and $100 \mu \mathrm{mol} \mathrm{L}^{-1}$ ) of 5-FU could induce apoptosis of the HeLa cells with normal cellular morphology; however, the higher concentration $\left(1,000 \mu \mathrm{mol} \mathrm{L}{ }^{-1}\right)$ of 5 -FU led to intensive cell death with fluorescence in almost all cells.

The HeLa (Caspase-3 biosensor) and MCF-7 (Caspase-3 biosensor) cells were exposed to different concentrations of adriamycin $\left(0.4,4\right.$, and $\left.40 \mu \mathrm{mol} \mathrm{L}{ }^{-1}\right)$ for $48 \mathrm{~h}$. The lowest concentration of adriamycin $\left(0.4 \mu \mathrm{mol} \mathrm{L}{ }^{-1}\right)$ induced obvious apoptosis in the HeLa (Caspase-3 biosensor) cells. The fluorescence positive cells were significantly increased at the concentration of $4 \mu \mathrm{mol} \mathrm{L}{ }^{-1}$ and decreased at the concentration of $40 \mu \mathrm{mol} \mathrm{L}{ }^{-1}$. The higher concentration of adriamycin could not trigger significant apoptosis in the HeLa cells (Figure 2B). The number of fluorescence-positive MCF-7 cells at a concentration of $4 \mu \mathrm{mol} \mathrm{L}{ }^{-1}$ was more than that at a concentration of $0.4 \mu \mathrm{mol} \mathrm{L}{ }^{-1}$, but the apoptosis- inducing ability of adriamycin on the MCF-7 cells was negligible compared with that on the HeLa cells. This result showed that using the proper concentration of adriamycin was very important for inducing apoptosis (Figure S4 in Supporting Information).

Docetaxel showed a prominent apoptosis-inducing activity against the HeLa (Caspase-3 biosensor) and the MCF-7 (Caspase- 3 biosensor) cells after $8 \mathrm{~h}$ of stimulation. Docetaxel induced apoptosis of HeLa (Caspase-3 biosensor) and MCF-7 (Caspase-3 biosensor) cells in a dose-dependent manner, and the fluorescence-positive cells increased with increasing concentrations of docetaxel (Figure 4C and D). Docetaxel has a better killing effect on the MCF-7 cells than that on the HeLa cells at the same drug concentration. A low concentration of docetaxel $\left(10 \mathrm{nmol} \mathrm{L}^{-1}\right)$ was sufficient to induce MCF-7 (Caspase-3 biosensor) cells apoptosis, and the higher concentration of docetaxel $\left(1000 \mathrm{nmol} \mathrm{L}^{-1}\right)$ almost killed all the cells.

We examined the apoptosis-inducing potential of three drugs, namely 5-FU, adriamycin, and docetaxel on the HeLa and MCF-7 cells. There were differences in the 5-FU-induced apoptosis between the HeLa and MCF-7 cells. The results indicated that 5-FU could effectively induce apoptosis of the HeLa cells, but it had no effect on the MCF-7 cells. Adriamycin also showed a better killing effect on the HeLa cells compared with that on the MCF-7 cells. However, docetaxel almost killed all the MCF-7 cells at the concentration of $1000 \mathrm{nmol} \mathrm{L}^{-1}$, but this effect was not seen in the HeLa cells. These results showed that the apoptosis of the HeLa and MCF-7 cells seems to largely depend on the type of cells and concentration of drugs. The present data suggest that our Caspase-3 biosensor could be useful for assessing apoptosis and provide a new approach for the screening of anti-tumor agents.

\section{The Caspase-3 biosensor platform applied for screening anti-Zika virus drugs}

The Zika virus remains a public health threat due to the 
A

$5-\mathrm{FU}$

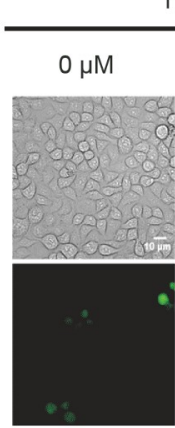

Hela (Caspase-3 biosensor)

$10 \mu \mathrm{M}$

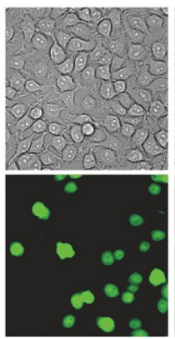

Hela (Caspase-3 biosensor)

C

Docetaxel

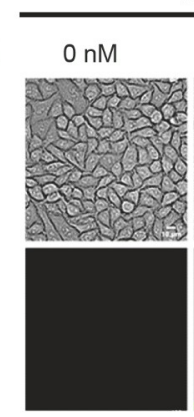

10

$100 \mu \mathrm{M}$

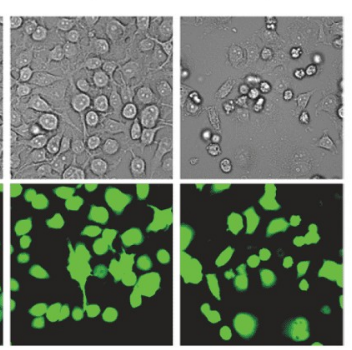

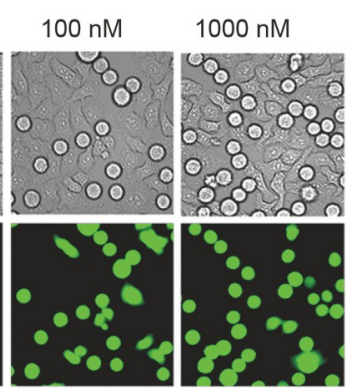

B

Adriamycin

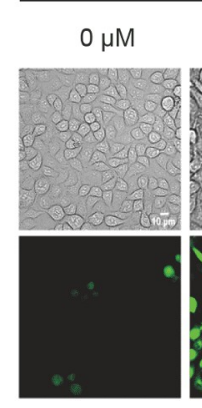

Hela (Caspase-3 biosensor)

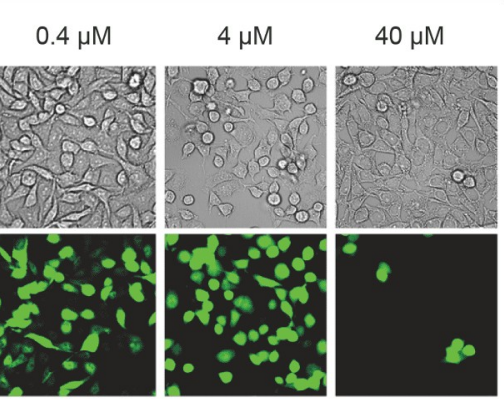

D

Docetaxel

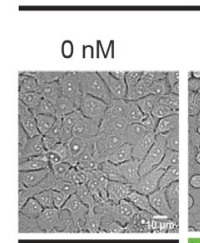

MCF-7 (Caspase-3 biosensor)

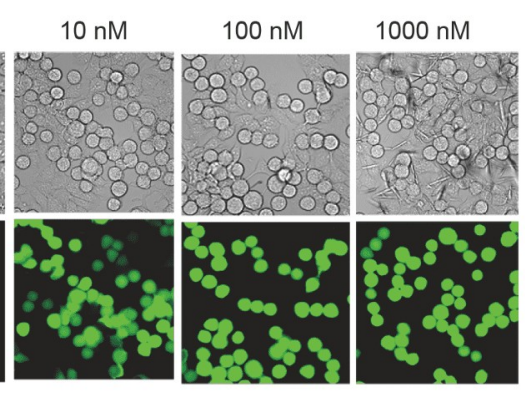

Figure 4 Apoptosis of tumor cells under different conditions. A, Fluorescence images of HeLa (Caspase-3 biosensor) cells were detected after treatment with different concentrations of 5-FU. B, Fluorescence images of HeLa (Caspase-3 biosensor) cells were detected after treatment with different concentrations of adriamycin. C, Fluorescence images of HeLa (Caspase-3 biosensor) cells were detected after treatment with different concentrations of docetaxel. D, Fluorescence images of MCF-7 (Caspase-3 biosensor) cells were detected after treatment with different concentrations of docetaxel.

congenital birth defects associated with it, which can impair cell cycle progression and provoke apoptosis (Lee et al., 2020; Yang et al., 2020). Currently, there are no approved vaccines or effective therapies to prevent or treat Zika virus infections. It has been demonstrated that the Zika virus induces apoptosis in A549, Vero, and human neural progenitor cells by increasing the activation of caspase 3 or 7 (Lee et al., 2020). To test whether the Zika virus could induce apoptosis of the HeLa (Caspase-3 biosensor) cells through the caspase3 pathway, the HeLa (Caspase-3 biosensor) cells were infected at different multiplicities of infection (MOI) of $0,0.1$, 0.5 , and $1 \mathrm{PFU} \mathrm{mL} \mathrm{L}^{-1}$. Subsequently, the fluorescence images were detected and processed by automated microscopy Cytation-3 after $36 \mathrm{~h}$ of infection. The HeLa (Caspase-3 biosensor) cells were highly permissive to the Zika virus infection at an MOI of 1 , and a few fluorescence positive cells were observed at an MOI of 0.01. There was a strong increase in the fluorescence signal with an increase of the MOI (Figure 5A and B). These results provided evidence that the Zika virus infection could trigger apoptosis of the HeLa cells through activation of caspase- 3 . The Caspase-3 biosensor may be an option to build a high-throughput screening platform for drug screening against the Zika virus.

We tested three drugs namely gemcitabine (Lee et al., 2017), remdesivir (Beigel et al., 2020) and obatoclax, (Wang et al., 2014) using this screening strategy, where the concentration of drugs was based on previous reports and con- siderations of their clinical use. First, we tested the toxicity of these drugs using different concentrations compared with the vehicle control (DMSO). The vehicle control (DMSO), along with gemcitabine and remdesivir, showed negligible toxicity, but obatoclax showed high toxicity on the HeLa cells. The low concentration of obatoclax $\left(5 \mu \mathrm{mol} \mathrm{L}{ }^{-1}\right)$ induced significant apoptosis; however, $50 \mu \mathrm{mol} \mathrm{L}{ }^{-1}$ obatoclax induced the formation of vesicles in cells, which is similar to the process of autophagy (Figure S5 in Supporting Information). The connection between autophagy and apoptosis has not been demonstrated adequately (Gump and Thorburn, 2011) and it is worth further study. The strong toxicity of obatoclax in the HeLa cells limited the application of the screening platform. Gemcitabine and remdesivir were used to pre-treat the HeLa (Caspase-3 biosensor) cells at concentrations of 1 and $10 \mu \mathrm{mol} \mathrm{L}{ }^{-1}$, respectively, for $1 \mathrm{~h}$ prior to infection with the Zika virus at an MOI of 0.5. Fluorescence intensity was detected after $36 \mathrm{~h}$ of infection. We found that both gemcitabine and remdesivir exhibited a potent antiviral effect at a concentration of $10 \mu \mathrm{mol} \mathrm{L}^{-1}$, and the infection rates of gemcitabine and remdesivir were $57 \%$ and $76 \%$, respectively, at $1 \mu \mathrm{mol} \mathrm{L}{ }^{-1}$ concentrations.

In summary, this study not only verified that the Zika virus can induce the apoptosis of HeLa cell, but also provided a new method for evaluation of antivirals. The fluorescent visual method for detection and quantification of infectious virus would provide an opportunity to develop new methods 
A

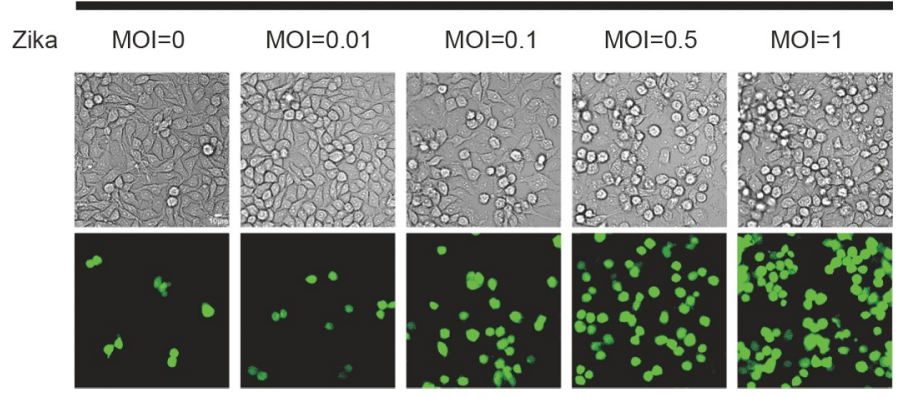

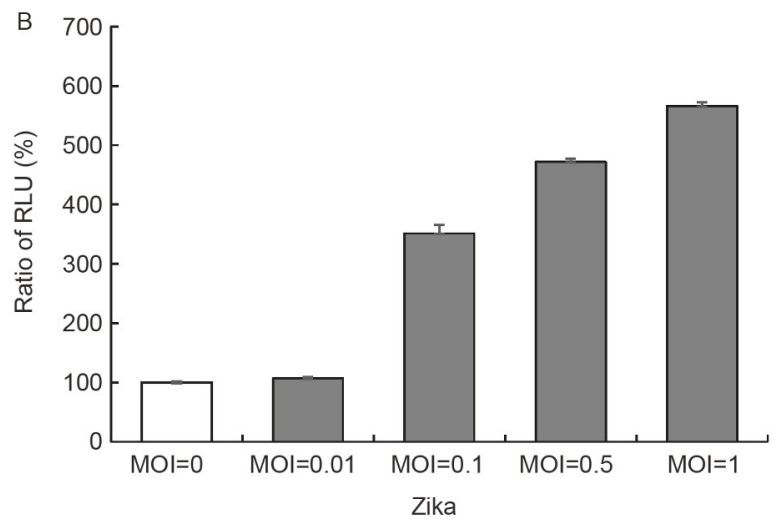

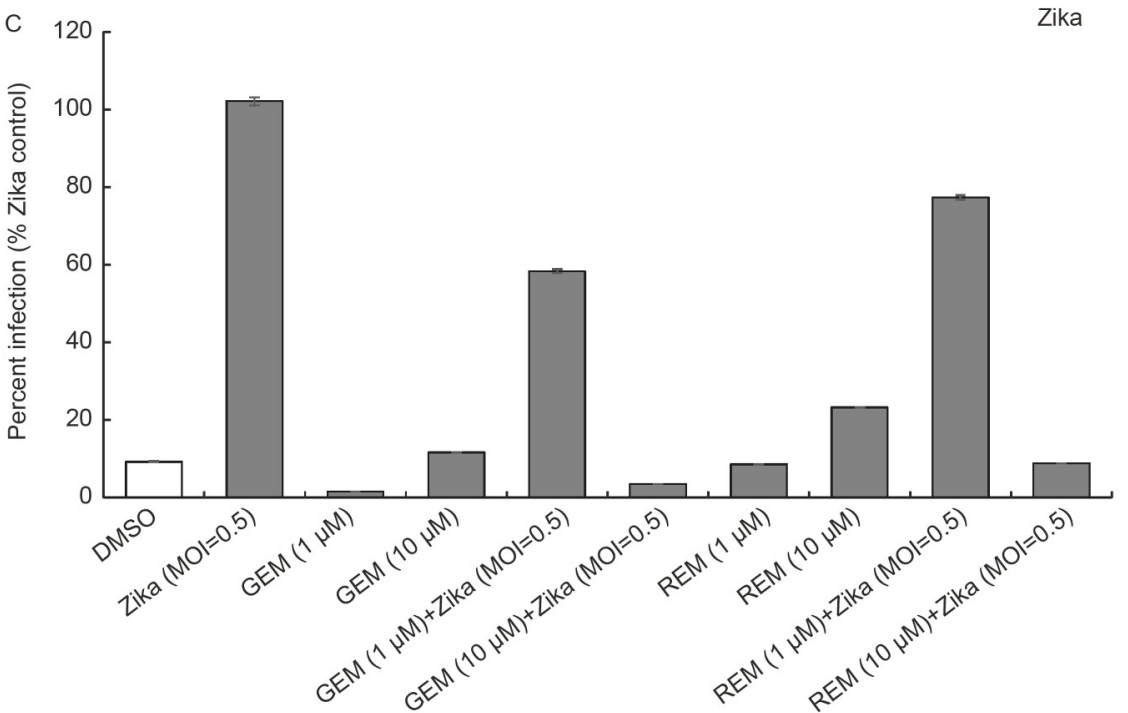

Figure 5 Evaluation of the Zika virus infection. A, Fluorescence images of HeLa (Caspase-3 biosensor) cells were detected after Zika virus infection for $36 \mathrm{~h}$ with different multiplicities of infection (MOI). B, Quantitative analysis of the effect of infection at different MOI. The relative fluorescence unit (RFU) is defined as the fluorescence intensity at different MOI divided by that obtained at an MOI of $0 . \mathrm{C}$, Quantitative analysis of fluorescence intensity of the HeLa (Caspase-3 biosensor) cells after different stimulation. DMSO is a vehicle control; GEM is gemcitabine and REM is remdesivir.

for fluorescence-activated cell sorting (FACS).

\section{DISCUSSION}

We developed a molecular biosensor using split-mNeonGreen2 that is selectively and specifically responsive to caspase-3 activity. The cyclization of the mNeonGreen2 leads to conformational distortion and prevents fluorescence emission. During apoptosis, activation of caspase-3 results in the site-specific cleavage of the DEVD sequence in the cyclic mNeonGreen2, leading to a fluorescence emission signal via reassembly of the $\mathrm{mNeonGreen} 2$ protein structure. The circular permutation sites and the length of the biosensor were optimized to minimize the background fluorescence and increase the specificity. Finally, we obtained a switch-on biosensor namely Caspase-3 biosensor. This biosensor has two key advantages compared with the existing counterparts. Firstly, the maturation time of the Caspase- 3 biosensor is about $80 \mathrm{~min}$, which is faster and rapid than the $90 \mathrm{~min}$ duration previously reported for FRET-based apoptosis
(Rehm et al., 2002) and the 400 min for FlipGFP (Zhang et al., 2019). Secondly, the caspase-3 sensor has very low background noise and a 10-fold enhanced fluorescence upon caspase- 3 activation, which is similar to the cyclic Luciferase sensor (Kanno et al., 2007) but without the requirement of any additional substrate for fluorescence emission.

We also used this biosensor to investigate the activation of caspase-3 after stimulation with 5-FU, adriamycin, and docetaxel. Among these drugs, docetaxel significantly increased the expression level of caspase- 3 within $8 \mathrm{~h}$, which is consistent as reported previously that docetaxel exhibited strong apoptotic induction effect both on HeLa cells and MCF-7 cells (Al-Abd et al., 2011). 5-FU and adriamycin induced the activation of caspase- 3 at $48 \mathrm{~h}$ in Hela cells, which is similar to the former studies (Brisdelli et al., 2016; Mavrikou et al., 2019). In contrast, the induction of caspase3 activity in MCF-7 cells by adriamycin was negligible relative to that in HeLa cells, while caspase- 3 in MCF-7 cells was not activated by 5 -FU stimulation. These results provide substantial evidence to support that the death of MCF-7 cells was mainly dependent on mitochondrial oxidative cytotoxi- 
city, while the caspase activity of 5-FU treated MCF-7 cells showed no significant change.

Interestingly, in our preliminary investigation, it was found that the Zika virus could induce production of caspase- 3 in Hela cells, leading to the apoptosis. Therefore, we hypothesized that assay of caspase-3 activated by Zika virus may help to screen the antiviral drugs. Using the biosensor, we examined three broad-spectrum antiviral drugs and found that gemcitabine and remdesivir could effectively and significantly inhibit Zika virus-induced apoptosis.

Caspases are a family with about 10 cysteine proteases that drive the cell death through substrate cleavage (Miller, 1997). Mostly the cell death requires more than one type of caspase. For example, the initiator caspases-2, -8, -9 and -10 activate the executioner caspase- $3,-6$, and -7 to direct the apoptosis (McIlwain et al., 2013). Monitoring the activation of the multiple caspases in the cell process is challenging but worthy to explore.

In conclusion, we constructed a genetically encoded cyclic $\mathrm{mNeonGreen} 2$ sensor to monitor the caspase-3 activity in living cells. The endogenous molecular biosensor can specifically and quantitatively measure the change of fluorescence following the activation of caspase-3, which enables high-throughput screening and analysis of anticancer as well as the antiviral drugs that cause apoptosis. The current protocol would enable us to develop a series of caspase biosensors by simply designing the corresponding cleavage site of the biosensor structure, which we believe will greatly deepen our understanding of the apoptosis mechanism and the molecular logic of caspase family, thereby improving the efficiency and efficacy of the clinical treatment of the diseases.

\section{MATERIALS AND METHODS}

\section{Mammalian cell lines and reagents}

HEK-293T (ATCC ${ }^{\circledR}$ CRL-11268), HeLa (ATCC ${ }^{\circledR}$ CCL-2 $^{\text {TM }}$ ), and MCF-7 (ATCC ${ }^{\circledR}$ CRL-3435 ${ }^{\mathrm{TM}}$ ) cells were maintained in our laboratory. Staurosporine (STS), N-benzyloxycarbonylVal-Ala-Asp (OMe) fluoromethyl ketone (Z-VAD-fmk), tumor necrosis factor- $\alpha$ (TNF- $\alpha)$, 5-fluorouracil (5-FU), cisplatin, docetaxel, adriamycin, gemcitabine, remdesivir, and obatoclax were purchased from Sigma, Germany.

\section{Plasmids}

The sequences of the PCR primers and the sequences encoding Npu DnaE intein were synthesized by Sango Biotechnology. Other biosensors were generated by PCR (PrimerSTAR Max, TaKaRa, Japan) and were cloned into a vector using the $\mathrm{pEASY}^{\circledR}$-Uni Seamless Cloning and Assembly Kit (TransGen Biotech, Beijing, China). DH5 $\alpha$, the
E. coli strain (TransGen Biotech, Beijing, China), was used as the bacterial host for the collection of all plasmids.

\section{Cell culture and transfection}

All the cells were maintained in DMEM (Gibco, USA) supplemented with 10\% fetal bovine serum (Gibco, USA) and $1 \%$ penicillin/streptomycin (Gibco, USA) at $37^{\circ} \mathrm{C}$ in an incubator with a humidified atmosphere of $5 \% \mathrm{CO}_{2}$ in air. Cells were transfected using Lipofectamine 3000 (Invitrogen, USA) in Opti-MEM (Invitrogen, USA) according to the manufacturer's recommended protocol.

\section{Generation of stable cells}

To generate stable cells, the plasmids plvx-Caspase- 3 biosensor and auxiliary plasmids were transfected into $293 \mathrm{~T}$ cells using Lipofectamine 3000 (Invitrogen, USA) when cells reached $50 \%-60 \%$ confluence, according to the manufacturer's protocol. The supernatant was then harvested and infected with 293T, HeLa, and MCF-7 cells. The cells were then sorted by puromycin, and the cells stably expressed Caspase-3 biosensor.

\section{Western blotting}

Cells were collected and lysed in RIPA (50 mmol L ${ }^{-1}$ Tris$\mathrm{HCl}, \mathrm{pH} 7.4,150 \mathrm{mmol} \mathrm{L}^{-1} \mathrm{NaCl}, 1 \%$ Triton $\mathrm{X}-100,1 \%$ sodium deoxycholate, $0.1 \%$ SDS) buffer containing cocktail (Sigma, USA), and sample loading was normalized according to BCA protein quantification. Stand sample was first separated by $12 \%$ sodium dodecyl sulfate (SDS)-polyacrylamide gel electrophoresis and transferred to polyvinylidene difluoride membranes (Millipore, Billerica, USA). Anti-mNeonGreen (rabbit, CST, \#53061), anti-caspase-3 (rabbit, CST, \#14220) and anti- $\beta$-tubulin (mouse, CST, \#86298) were used as primary antibodies. Then, the blots were incubated with horseradish peroxidase-conjugated secondary antibodies (ABclonal, AS014 or AS003). The immunoblots were imaged with clarity western ECL substrate (Bio-Rad, USA).

\section{Virus stocks}

The Zika virus strain (PRVABC59) $\left(\right.$ ATCC $^{\circledR}$ VR-1843 ${ }^{\text {TM}}$ ) was purchased from ATCC, USA. Zika virus was propagated in $\mathrm{C} 6 / 36$ cells and titrated on Vero cells.

\section{Imaging of cultured cells}

For live-cell imaging, the cells were plated on six-well plates and stimulated under desired conditions. The cells were then maintained in growth medium and were imaged by Cytation- 
3 (BioTek Instruments, USA) at different times. All the fluorescence intensity at different conditions was measured on a 96-well plate by Cytation-3 (BioTek Instruments, USA).

\section{Data and statistical analysis}

All data were collected in triplicates or more, and are presented as the mean \pm standard deviation (SD).

\section{Compliance and ethics The author(s) declare that they have no conflict of interest.}

\begin{abstract}
Acknowledgements This work was supported by the National Natural Science Foundation of China (21890743), National Key Research and Development Program of China (2017YFA0205500, 2018YFA0902702), the Strategic Priority Research Program of the Chinese Academy of Sciences, China (XDB29050100). We thank Yu Hou and Liu Song for their helpful discussions.
\end{abstract}

\section{References}

Al-Abd, A.M., Mahmoud, A.M., El-Sherbiny, G.A., El-Moselhy, M.A., Nofal, S.M., El-Latif, H.A., El-Eraky, W.I., and El-Shemy, H.A. (2011). Resveratrol enhances the cytotoxic profile of docetaxel and doxorubicin in solid tumour cell lines in vitro. Cell Proliferation 44, 591-601.

Bardet, P.L., Kolahgar, G., Mynett, A., Miguel-Aliaga, I., Briscoe, J., Meier, P., and Vincent, J.P. (2008). A fluorescent reporter of caspase activity for live imaging. Proc Natl Acad Sci USA 105, 13901-13905.

Beigel, J.H., Tomashek, K.M., Dodd, L.E., Mehta, A.K., Zingman, B.S., Kalil, A.C., Hohmann, E., Chu, H.Y., Luetkemeyer, A., Kline, S., et al. (2020). Remdesivir for the treatment of COVID-19-final report. N Engl J Med 383, 1813-1826.

Blum, R.H., and Carter, S.K. (1974). Adriamycin. Ann Intern Med 80, 249 259.

Brisdelli, F., Perilli, M., Sellitri, D., Bellio, P., Bozzi, A., Amicosante, G., Nicoletti, M., Piovano, M., and Celenza, G. (2016). Protolichesterinic acid enhances doxorubicin-induced apoptosis in hela cells in vitro. Life Sci 158, 89-97.

Conrad, C., Di Domizio, J., Mylonas, A., Belkhodja, C., Demaria, O., Navarini, A.A., Lapointe, A.K., French, L.E., Vernez, M., and Gilliet, M. (2018). TNF blockade induces a dysregulated type I interferon response without autoimmunity in paradoxical psoriasis. Nat Commun $9,1$.

Cotter, T.G. (2009). Apoptosis and cancer: The genesis of a research field. Nat Rev Cancer 9, 501-507.

Galluzzi, L., Vitale, I., Abrams, J.M., Alnemri, E.S., Baehrecke, E.H., Blagosklonny, M.V., Dawson, T.M., Dawson, V.L., El-Deiry, W.S., Fulda, S., et al. (2012). Molecular definitions of cell death subroutines: recommendations of the nomenclature committee on cell death 2012. Cell Death Differ 19, 107-120.

Guerreiro, M.R., Freitas, D.F., Alves, P.M., and Coroadinha, A.S. (2019). Detection and quantification of label-free infectious adenovirus using a switch-on cell-based fluorescent biosensor. ACS Sens 4, 1654-1661.

Gump, J.M., and Thorburn, A. (2011). Autophagy and apoptosis: What is the connection? Trends Cell Biol 21, 387-392.

Hellwig, C.T., Kohler, B.F., Lehtivarjo, A.K., Dussmann, H., Courtney, M. J., Prehn, J.H.M., and Rehm, M. (2008). Real time analysis of tumor necrosis factor-related apoptosis-inducing ligand/cycloheximideinduced caspase activities during apoptosis initiation. J Biol Chem 283, 21676-21685.

Hengartner, M.O. (2000). The biochemistry of apoptosis. Nature 407, $770-$ 776.

Jacobson, M.D., Weil, M., and Raff, M.C. (1997). Programmed cell death in animal development. Cell 88, 347-354.
Kanno, A., Umezawa, Y., and Ozawa, T. (2009). Detection of apoptosis using cyclic luciferase in living mammals. Bioluminescence, 105-114.

Kanno, A., Yamanaka, Y., Hirano, H., Umezawa, Y., and Ozawa, T. (2007). Cyclic luciferase for real-time sensing of caspase-3 activities in living mammals. Angew Chem Int Ed 46, 7595-7599.

Lee, J.K., Kim, J.A., Oh, S.J., Lee, E.W., and Shin, O.S. (2020). Zika virus induces tumor necrosis factor-related apoptosis inducing ligand (trail)mediated apoptosis in human neural progenitor cells. 9, 2487.

Lee, K., Kim, D.E., Jang, K.S., Kim, S.J., Cho, S., and Kim, C. (2017). Gemcitabine, a broad-spectrum antiviral drug, suppresses enterovirus infections through innate immunity induced by the inhibition of pyrimidine biosynthesis and nucleotide depletion. Oncotarget 8, $115315-115325$.

Martin, J.C., Chang, C., Boschetti, G., Ungaro, R., Giri, M., Grout, J.A., Gettler, K., Chuang, L.S., Nayar, S., Greenstein, A.J., et al. (2019). Single-cell analysis of Crohn's disease lesions identifies a pathogenic cellular module associated with resistance to anti-TNF therapy. Cell 178, 1493-1508.e20.

Mavrikou, S., Tsekouras, V., Karageorgou, M.A., Moschopoulou, G., and Kintzios, S. (2019). Detection of superoxide alterations induced by 5fluorouracil on HeLa cells with a cell-based biosensor. Biosensors 9, 126.

McIlwain, D.R., Berger, T., and Mak, T.W. (2013). Caspase functions in cell death and disease. Cold Spring Harb Perspect Biol 5, a008656.

Miller, D.K. (1997). The role of the caspase family of cysteine proteases in apoptosis. Semin Immunol 9, 35-49.

Nasu, Y., Asaoka, Y., Namae, M., Nishina, H., Yoshimura, H., and Ozawa, T. (2016). Genetically encoded fluorescent probe for imaging apoptosis in vivo with spontaneous GFP complementation. Anal Chem 88, 838844.

Nicholls, S.B., Chu, J., Abbruzzese, G., Tremblay, K.D., and Hardy, J.A. (2011). Mechanism of a genetically encoded dark-to-bright reporter for caspase activity. J Biol Chem 286, 24977-24986.

Nijhawan, D., Honarpour, N., and Wang, X. (2000). Apoptosis in neural development and disease. Annu Rev Neurosci 23, 73-87.

Pham, V.C., Pitti, R., Anania, V.G., Bakalarski, C.E., Bustos, D., Jhunjhunwala, S., Phung, Q.T., Yu, K., Forrest, W.F., Kirkpatrick, D. S., et al. (2012). Complementary proteomic tools for the dissection of apoptotic proteolysis events. J Proteome Res 11, 2947-2954.

Porter, A.G., and Jänicke, R.U. (1999). Emerging roles of caspase-3 in apoptosis. Cell Death Differ 6, 99-104.

Rechsteiner, M., and Rogers, S.W. (1996). Pest sequences and regulation by proteolysis. Trends Biochem Sci 21, 267-271.

Rehm, M., Dussmann, H., Janicke, R.U., Tavare, J.M., Kogel, D., and Prehn, J.H.M. (2002). Single-cell fluorescence resonance energy transfer analysis demonstrates that caspase activation during apoptosis is a rapid process. J Biol Chem 277, 24506-24514.

Sara, J.D., Kaur, J., Khodadadi, R., Rehman, M., Lobo, R., Chakrabarti, S., Herrmann, J., Lerman, A., and Grothey, A. (2018). 5-fluorouracil and cardiotoxicity: a review. Ther Adv Med Oncol 10, 175883591878014.

Shaner, N.C., Lambert, G.G., Chammas, A., Ni, Y., Cranfill, P.J., Baird, M. A., Sell, B.R., Allen, J.R., Day, R.N., Israelsson, M., et al. (2013). A bright monomeric green fluorescent protein derived from branchiostoma lanceolatum. Nat Methods 10, 407-409.

Stefanis, L., and Rideout, H. (2001). Caspase inhibition: a potential therapeutic strategy in neurological diseases. Histol Histopathol 16, 895908.

Sun, C., Zhu, L., Zhang, C., Song, C., Wang, C., Zhang, M., Xie, Y., and Schaefer Iii, H.F. (2018). Conformers, properties, and docking mechanism of the anticancer drug docetaxel: DFT and molecular dynamics studies. J Comput Chem 39, 889-900.

Valentin, G., Verheggen, C., Piolot, T., Neel, H., Coppey-Moisan, M., and Bertrand, E. (2005). Photoconversion of YFP into a CFP-like species during acceptor photobleaching FRET experiments. Nat Methods 2, 801.

Wang, G., Chen, S., Edwards, H., Cui, X., Cui, L.I., and Ge, Y. (2014). Combination of chloroquine and GX15-070 (obatoclax) results in 
synergistic cytotoxicity against pancreatic cancer cells. Oncol Rep 32, 2789-2794.

Xu, Z., Song, Y., and Wang, F. (2020). Rational design of genetically encoded reporter genes for optical imaging of apoptosis. Apoptosis 25 , 459-473.

Yang, S., Gorshkov, K., Lee, E.M., Xu, M., Cheng, Y.S., Sun, N., Soheilian, F., de Val, N., Ming, G., Song, H., et al. (2020). Zika virus-induced neuronal apoptosis via increased mitochondrial fragmentation. Front Microbiol 11, 598203.
Ivol(Yongxin Zhaoet al.), . Zhang, J., Wang, X., Cui, W., Wang, W., Zhang, H., Liu, L., Zhang, Z., Li, Z., Ying, G., Zhang, N., et al. (2013). Visualization of caspase-3-like activity in cells using a genetically encoded fluorescent biosensor activated by protein cleavage. Nat Commun 2157.

Zhang, Q., Schepis, A., Huang, H., Yang, J., Ma, W., Torra, J., Zhang, S.Q., Yang, L., Wu, H., Nonell, S., et al. (2019). Designing a green fluorogenic protease reporter by flipping a beta strand of GFP for imaging apoptosis in animals. J Am Chem Soc 141, 4526-4530.

\section{SUPPORTING INFORMATION}

The supporting information is available online at https://doi.org/10.1007/s11427-021-1986-7. The supporting materials are published as submitted, without typesetting or editing. The responsibility for scientific accuracy and content remains entirely with the authors. 